\title{
DU LABORATOIRE AU CÉDÉROM : EXPÉRIENCE SIMULÉE ET CONSTRUCTION DE CONNAISSANCES ${ }^{1}$
}

Annette Béguin et Bénédicte Amougou²

Aux savoirs quotidiens informels et ancrés dans l'expérience ordinaire, Lev Vygotski ${ }^{3}$ oppose les savoirs scientifiques, formalisés et susceptibles de verbalisation. Toute acquisition d'un savoir scientifique suppose la remise en question de représentations mentales antérieures issues des savoirs quotidiens. La médiation pédagogique, selon Vygotski, conduit les élèves à cette remise en question par l'expérimentation d'une part et par la verbalisation d'autre part. Que se passe-t-il si l'on remplace la démarche classique d'apprentissage par l'usage d'un produit multimédia ? Jusqu'à quel point l'introduction du média, qui a sa propre "forme", modifie-t-elle la construction du savoir?

Les discours sur les nouvelles technologies à l'école revêtent souvent un caractère promotionnel. Les produits multimédias sont, dit-on, "ludiques et interactifs", ce qui devrait garantir leur succès

1 Une partie de ce texte reprend une communication effectuée à Louvain-la-Neuve dans le cadre du colloque intitulé "Savoirs formels, savoir informels", organisé par le GReMS en décembre 2000.

2 Université de Lille 3.

${ }^{3}$ L. VyGotski, Pensée et langage, Paris, Messidor/Editions sociales, 1985. 
auprès du jeune public et, partant, leur efficacité pédagogique ${ }^{1}$. Une réflexion plus poussée sur les usages devrait permettre de dépasser cette manifestation de l'idéologie dominante en matière de technique et de nouveauté 2 . Il s'agit d'étudier les produits multimédias dans leurs particularités et en relation avec les formes d'appropriation spécifiques auxquelles ils donnent lieu. A cette condition, il sera possible de réfléchir à leur insertion dans les dispositifs pédagogiques visant à la transmission des connaissances.

C'est dans cette perspective que nous nous sommes intéressés aux cédéroms culturels, qui ont la faveur des CDI d'établissements scolaires et plus particulièrement à la simulation d'expériences scientifiques à l'écran. Notre hypothèse est que l'énergie mentale que le sujet développe dans ce genre de simulation est en grande partie mobilisée pour résoudre des problèmes liés à la virtualisation de l'expérience, organisée par le dispositif sémiotique, et non par l'expérience elle-même.

L'observation du "simulateur de teinture en cuve", que nous relatons ci-dessous, fait partie d'un ensemble de recherches menées à Lille 3 sur les écrits d'écran et de réseaux. Elle doit être prolongée et relativisée. Malgré ses limites, elle nous a permis d'interroger le rapport entre la représentation du monde à l'écran, la représentation scientifique que le dispositif devrait permettre de construire et les représentations du monde ordinaire que l'usager réinvestit lorsqu'il utilise le produit.

\section{Le protocole d'observation}

Nous avons choisi comme objet le cédérom intitulé "Le secret des couleurs"3. Il a retenu notre attention par la publicité qui en était faite : revue de presse élogieuse et diffusion d'une version de

1 Voir sur ce point: A. BÉGuIN, "Entre interactivité et médiation : quelques interrogations sur les usages des nouveaux médias dans l'enseignement", in FADBEN, Pour un élève info-zappeur ou info-lettré ? : 5ème congrès des enseignants documentalistes de l'Éducation nationale, Bordeaux, 26-28 mars 1999, Paris, Nathan, 2000, pp. 67-68 et pp. 107-115.

2 On se reportera fort utilement sur ce point à Y. JEANNERET, Y a-t-il (vraiment) des technologies de l'information?, Lille, Presses Universitaires du Septentrion, 2000, Chap. 2, "Nouvelles technologies de l'information": une expression mal formée, pp. 41-64.

3 Fr. Pignet, Le secret des couleurs, Chimagora, Carré Multimédia, 1997. 
démonstration avec la revue de vulgarisation pour adolescent Eurêka. Ce cédérom est décrit comme étant "un passionnant voyage à travers l'univers des couleurs".

Notre étude porte sur une séquence interactive intitulée "teintez votre jean". Il s'agit ici d'acquérir des connaissances sur la chimie et les procédés de teinture. Une des raisons qui a guidé notre choix vers cette partie est qu'il s'agit d'une séquence "fermée", sans lien avec le reste du cédérom, ce qui facilite l'observation des usages. D'autre part, nous avons été attirées par la proposition alléchante qui figure au dos du produit : "teintez vous-même votre jean grâce à un véritable simulateur de teinture en cuve à l'indigo !'. Le choix du jean, objet emblématique des adolescents, indique quelle est la cible visée. Quant à l'insistance pour le moins curieuse sur le "véritable simulateur", elle nous ramène au rêve de la simulation parfaite dont l'avatar ultime serait l'immersion dans un univers à trois dimensions propre à fasciner les créateurs de science-fiction et à rendre les chercheurs sceptiques'. A la fin de l'apprentissage, le sujet devrait pouvoir réaliser l'expérience "en vrai".

Nous avons emprunté les modes d'observation que nous avons retenus à la psychologie cognitive ${ }^{2}$. Après avoir fait une analyse détaillée du cédérom, nous avons filmé l'utilisation de la séquence interactive par des utilisateurs de profils différents, puis les utilisateurs ont visualisé et commenté ce film en utilisant à leur gré la télécommande du magnétoscope. Cette visualisation a elle-même été filmée. Nous avons d'autre part procédé, entre la phase de manipulation à l'écran et la phase de commentaire, à un questionnaire évaluatif et à un test immédiat de mémorisation de la séquence. Nous disposons donc de données sur la manipulation elle-même, au plan gestuel et verbal, et de données sur la manière dont elle a été vécue et retenue, c'est-à-dire sur les représentations du dispositif et de la tâche que les utilisateurs ont élaborées au fil de l'action.

1 On se référera en particulier au film Existenz du réalisateur David Kronenberg. Le caractère utopique de l'illusion parfaite de réalité donnée par la troisième dimension a été très bien analysé par Igor Babou : I. BABOU, " $L$ 'absence de cadre comme utopie des «réalités virtuelles»", Champs Visuels, n 12-13, avril 1999, pp. 164-172.

2 J.-M. Hoc, "La verbalisation provoquée pour l'étude du fonctionnement cognitif", Psychologie française, $\mathrm{n}^{\circ} 29,1984$, pp. 231-234. J.-C. SPERANDIO, La psychologie en ergonomie, Paris, PUF, 1980. A. BORZEIX, "Comment observer l'interprétation", in A. BORzeIX, A. Bouvier, P. PHARo, Sociologie de la connaissance: nouvelles approches cognitives, Paris, CNRS éditions, 1998. 
Nous nous sommes efforcées de différencier les profils d'utilisateurs, en fonction de leurs connaissances antérieures de la chimie ou de l'outil informatique. Le dispositif complet, très coûteux en temps (en moyenne deux heures de passation par personne), n'a jusqu'ici été appliqué qu'à cinq personnes. Les bandes vidéo ont été intégralement décryptées. Nous avons effectué une analyse de contenu sur la transcription en prenant comme catégories les obstacles rencontrés, qu'ils soient ou non surmontés.

Sur la base des premiers résultats, nous avons mis au point une grille permettant d'effectuer des observations directes plus ciblées, tout en maintenant la partie écrite du protocole (questionnaire évaluatif et test de mémorisation). Cette technique, plus légère, rend possible la vérification de nos hypothèses sur un plus grand nombre d'utilisateurs' ${ }^{1}$.

Au fil de notre démarche, nous avons tenté de mettre en relation les données issues de l'analyse sémiotique du produit et les observations sur les usages de ce produit par des utilisateurs, si possible représentatifs des publics potentiels. Le sémioticien considère en effet l'interface comme le produit d'une énonciation dont elle porte la trace. Elle porte en creux la figure du "producteur modèle" et de "l'utilisateur modèle" tels qu'Umberto Eco les a définis à propos du texte de fiction dans Lector in fabula. Le cogniticien s'intéresse, lui, à l'utilisateur réel qui affronte un problème et aux stratégies qu'il va développer pour le résoudre. C'est au carrefour de la sémiotique et de la pragmatique cognitive que nous tentons de nous situer : il s'agit de réconcilier une approche des problèmes de lecture par l'objet avec une approche des problèmes de lecture par le sujet.

\section{Un univers sémiotique complexe, qui transforme le rapport à l'espace et au temps}

Dans les produits multimédias, la représentation du Monde au moyen d'un système de signe change en profondeur non seulement le

\footnotetext{
1 Nos résultats devront encore être relativisés par des observations complémentaires : - l'observation similaire d'autres séquences de simulation ;

- une observation de type ethnologique qui permettrait de dépasser le cadre de la séquence fermée et de prendre en compte les motivations de chaque utilisateur pour analyser sa démarche.
} 
rapport à la perception, mais aussi le rapport à l'action. Le mode de représentation, en effet, peut relever de l'image du son et du langage, mais l'interactivitél fait intervenir un niveau supplémentaire, qui relève de la programmation ${ }^{2}$. Par interactivité nous entendrons ici la relation entre l'action de l'utilisateur et l'opération de la machine déclenchée en retour.

Dans la simulation d'expérience, les paramètres spatio-temporels qui servent de repères lorsqu'il s'agit de maîtriser l'environnement naturel sont transformés. Le sujet est plongé dans un univers complexe au plan sémiotique qui, par les rapports d'analogie qu'il entretient avec le monde réel, fonctionne comme un leurre déstabilisant.

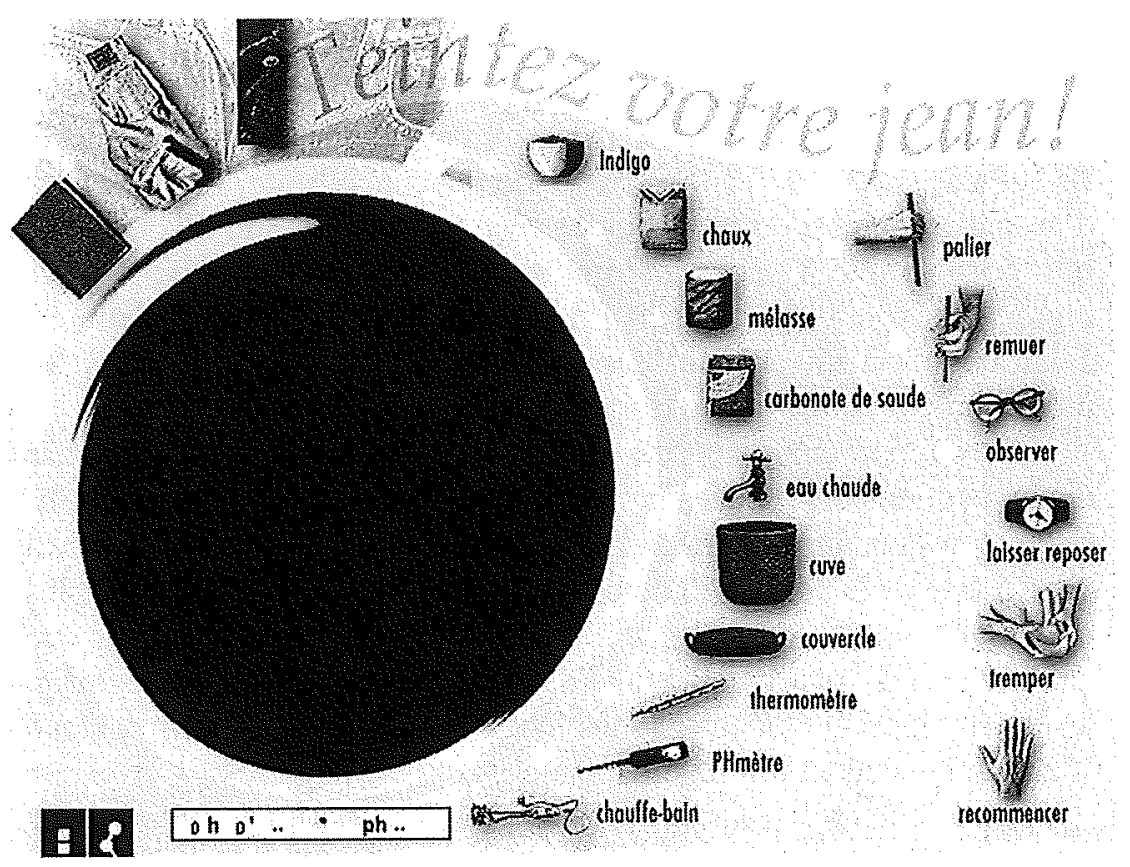

1 A. BÉGUIN, op. cit.

2 Y. JeAnNERET, E. Souchier, "Pour une poétique de l'écrit d'écran", Xoana, n 6 , 1999. 


\subsection{Incertitudes spatiales introduites par le système de signes $\grave{a}$ l'écran}

Les objets, tout d'abord, perdent la relation au contexte dans lequel ils sont d'ordinaire perçus. Dans le "simulateur de teinture en cuve", selon les habitudes du style "look and feel" instauré dans le documentaire par l'éditeur David Kindersley, ils sont représentés par des dessins détourés, ombrés et insérés sur un fond très clair, constitué par la photographie d'un jean. Cette décontextualisation s'accompagne d'un calibrage qui leur fait perdre leur échelle relative. Ainsi, l'expérience de teinture repose sur la détermination de quantités, or contenants et contenus perdent leurs proportions : la cuve est plus petite que les paquets et ressemble plus à un gobelet.

Les signes calibrés sont alignés selon deux demi-cercles. L'analogie avec la lecture alphabétique s'instaure naturellement ${ }^{1}$. Les utilisateurs commencent donc par les objets situés en haut à gauche. Ils suivent l'ordre de haut en bas et de gauche à droite. Ils imaginent d'autre part que cet ordre coïncide avec une planification d'actions, ce qui correspond à leurs habitudes en matière de cuisine: les ingrédients sont disposés dans l'ordre de la recette. Or ici l'ordre est aléatoire, d'où un premier déphasage qui intervient chez tous les utilisateurs, d'entrée de jeu trompés dans leurs attentes.

Par rapport aux objets du monde ordinaire, les objets à l'écran se distinguent également par une permanence incertaine. Ainsi, les utilisateurs ont tendance, au départ, à confondre le rond noir qui apparaît au début avec le dessus d'une cuve. Ils cherchent donc à y mettre les ingrédients, et ne songent pas à y placer...la cuve ! Celle-ci est vue tantôt de profil, tantôt de dessus (dans la fonction "observer"). Enfin, les objets figurent également sous forme d'un "fantôme" noir et blanc à leur emplacement initial une fois qu'ils ont été déplacés.

Un même objet peut être présent plusieurs fois à l'écran avec une fonctionnalité différente. Ainsi, le jean est présent dans la fonction "tremper", qui est activable ; mais il figure aussi en haut à gauche de l'écran et, à cet endroit, il ne peut être déplacé. Certains utilisateurs tentent en vain de l'activer en multipliant les clics. Autre exemple : la

1 A.-M. Christin, L'image écrite : ou la déraison graphique, Paris, Flammarion, 1995. 
main figure le pointeur qui sert à désigner ou à déplacer ; elle est attachée à certains objets comme partie du corps sensée agir ("palier", "remuer", "tremper"). Il en résulte deux niveaux d'iconicité : "je prends une main avec la main". Enfin, par catachrèse, la main signifie aussi la fonction "recommencer" (passer la main ou stop !).

Si la cohérence d'un système de signes, sa grammaire, en garantit la lisibilité, le produit qui nous sert d'exemple présente quelques défauts. Devant la régularité du dispositif graphique, l'utilisateur présuppose une homogénéité de statut entre les objets présents à l'écran. Or certains objets sont mobiles (thermomètre) et d'autres non mobiles (montre, lunettes) alors qu'ils semblent alignés sur le même paradigme. Certains sont assortis d'une main et d'autres non. Certaines icônes sont des représentations directes de la réalité, alors que d'autres ont une valeur métonymique. Elles peuvent cependant être prises au premier degré si on ne lit pas le mot qui les accompagne. Ainsi les lunettes (et non l'œil) sont mises pour "observer", la montre (sans main) et son tic-tac pour "laisser reposer".

Les objets sont donc reliés par une cohérence graphique qui n'a rien à voir avec la cohérence d'usage qu'ils entretiennent dans la réalité.

\subsection{Limites imposées par la programmation et temporalité}

Outre la complexité du système de signes utilisé, la gestion informatisée de son fonctionnement introduit des germes d'incertitude. La représentation du temps (ordre, durée, vitesse, accélération...) en est modifiée en profondeur. L'outil informatique génère des contraintes nouvelles par rapport à l'expérience en réel.

La référence implicite en matière de conduite naturelle est ici le script de la recette de cuisine, souvent évoqué par les utilisateurs. Une des utilisatrices parle même de "soupe". Or, le déroulement est indiqué dans un livre qui devrait servir de guide principal : la simultanéité des lectures (recette/écran) est impossible. Le livre ouvert cache, en effet, tout l'espace de l'expérience. On ne peut pas imprimer la page en compensation, ce que permettent certains cédéroms. On ne peut pas non plus gérer conjointement deux actions parfaitement compatibles dans la vie quotidienne comme verser des ingrédients et mélanger. Une utilisatrice en a été perturbée : 
Oui, alors là, lui, il me disait "remuer en ajoutant", mais tu ne peux pas faire les deux. Tu vois, si tu rajoutes l'ingrédient, il ne remue plus. Il dit "remuer en ajoutant", ouais, enfin, "ajouter en remuant"... En fait, tu ne peux plus remuer quand tu ajoutes, tu ne remues plus.

On ne peut pas, enfin, contrôler finement les quantités. Les choix sont imposés à l'utilisateur, or ils ne correspondent pas aux quantités prescrites dans le livre. Une des instructions mentionne : dissoudre la poudre d'indigo $(7 \mathrm{~g} / 1)$. Après calcul, on ne trouve pas la quantité demandée dans les choix possibles, ce qui génère souvent des erreurs de dosage. Ces erreurs ne peuvent pas être corrigées. Si l'opération déclenchée ne convient pas, on ne peut pas revenir en arrière. Un clic de trop et il faut reprendre tout à zéro.

La durée est l'élément le plus difficile à représenter. Quand le présentateur annonce que l'expérience va durer 36 heures, certains utilisateurs s'esclaffent. Une utilisatrice s'inquiète même :

Je me suis dit, je vais devoir rester 36 heures. Je me suis dit que ça faisait peut-être un petit peu long !

Mais on s'aperçoit très vite que cette durée est fictive et qu'on n'a pas de prise sur elle. Il est impossible, par exemple, de reproduire des processus naturels comme "laisser refroidir" lorsqu'on s'aperçoit que la solution est trop chaude.

Les utilisateurs sont perturbés par les indications temporelles qui renvoient tantôt au temps de la simulation, tantôt au temps de l'expérience "en réel", tantôt au temps de calcul de la machine. Ainsi, les utilisateurs ont-ils été surpris par le temps correspondant à la fonction "tremper", tantôt très bref, tantôt très long. En fait, si l'utilisateur a procédé à de nombreuses manipulations avant d'activer cette fonction, la machine procède à un long calcul pour sortir le graphe du résultat. La sortie est au contraire très rapide s'il n'y a que quelques manipulations.

Enfin, la perception de l'écoulement du temps est toujours double. L'utilisateur est pris entre le temps réel de son parcours dans le cédérom et le temps symbolisé de l'expérience simulée, inscrit en bas de l'écran sous forme de chiffres dans un cartouche. Il n'y a pas de relation continue entre les deux temporalités qui ne sont pas proportionnelles. Ce temps double est difficile à gérer au plan conceptuel et demande un calcul prévisionnel en ce qui concerne le 
temps de l'expérience. Le temps symbolisé permet des suspensions réflexives, mais il introduit aussi, parfois, un sentiment d'urgence perturbateur qui n'a rien à voir avec l'expérience elle-même : les utilisateurs ont le sentiment de "devoir faire quelque chose" alors même qu'ils ne sont pas certains de l'action à entreprendre.

L'analyse du dispositif sémiotique met en évidence des difficultés potentielles de lecture. Elles sont sans doute très flagrantes dans le cédérom que nous avons choisi comme objet d'étude. Cependant la nature même de ces difficultés est inhérente au média : nous avons affaire à des représentations d'objets qui sont elles-mêmes des objets. L'utilisateur est donc contraint d'engager deux niveaux d'action : l'action sur les objets du monde simulé, qui le ramène à sa connaissance ordinaire, et l'action sur les objets à l'écran, qui sont des symboles mais qui sont aussi des objets sur lesquels on peut aussi agir, quoique différemment. Le caractère analogique de la représentation génère inévitablement de la confusion, au moins au premier abord. L'utilisateur croit reconnaître un environnement familier, or il faut apprendre comment fonctionne le système des signes pour pouvoir réaliser l'expérience.

\section{Utilisations des ressources cognitives et apprentissages}

Dans cet univers incertain et déstabilisant parce qu'il ressemble à l'univers ordinaire sans en avoir toutes les caractéristiques, l'utilisateur peut-il construire un savoir transférable ? Son énergie risque d'être entièrement captée par la nécessité de se donner d'abord des repères dans le monde fictif qui lui est proposé. Pire, il est amené à "désapprendre" ses repères ordinaires.

A quelles formes de connaissances avons-nous affaire dans de tels environnements? Les observations que nous avons menées tendent à confirmer la théorie de la cognition incarnée ou énaction développée par Francisco Varela ${ }^{1}$. Varela s'appuyant sur les travaux de Merleau-Ponty et de Mark Johnson, affirme l'importance de l'expérience corporelle dans la cognition. Il propose de traiter le savoir-faire :

1 Fr. VAREla, E. Thompson, E. Rosch, L'inscription corporelle de l'esprit : sciences cognitives et expérience humaine, Paris, Éd. du Seuil, 1993, Chap. 8 : L'énaction : cognition incamée, pp. 207-212. 
Non comme un artefact résiduel qui pourra être progressivement éliminé par la découverte de règles de plus en plus élaborées, mais en fait, comme l'essence même de la cognition créatrice.

Dans ces conditions, l'expérience accumulée au cours de nombreuses situations concrètes est constitutive du savoir :

La connaissance est le résultat d'une interprétation permanente qui émerge de nos capacités de compréhension. Ces capacités s'enracinent dans les structures de notre corporéité biologique, mais elles sont vécues et éprouvées à l'intérieur d'un domaine d'action consensuelle et d'histoire culturelle.

Ces connaissances, dont il affirme ainsi le caractère à la fois corporel et social, nous permettent, dit-il, de "posséder un monde".

De fait, l'utilisateur du simulateur de teinture utilise son savoir ordinaire pour pénétrer l'univers qui lui est proposé. Il répond aux difficultés qu'il rencontre par des routines qu'il a intégrées dans sa vie quotidienne, ainsi qu'il le ferait dans une situation de découverte empirique. Il est encouragé à cela par l'organisation sémiotique et la présentation publicitaire du document : le monde virtuel est désigné comme le reflet fidèle du monde réel, au point que des apprentissages construits dans ce monde pourraient s'appliquer au monde réel. "Teintez votre jean..." indique la publicité du cédérom. En fait, il n'est pas évident qu'un tel transfert soit possible.

Nier la dimension corporelle de la cognition peut conduire à méconnaître les difficultés liées à l'apprentissage par simulation. Comme l'indique Varela :

Quand les domaines des tâches à effectuer sont étendus des micro-mondes artificiels au monde en général, il n'est pas évident que nous puissions jamais spécifier ce qui compte comme objet indépendamment du type d'action qu'il s'agit d'accomplir.

\subsection{Négation dans l'action des savoirs incorporés}

Les utilisateurs du "simulateur de teinture en cuve" sont tenus par deux types de contraintes opposées : ils sont obligés de mobiliser leur connaissance du monde pour faire face à une situation non connue, mais ils doivent en même temps se départir d'un certain 
nombre de savoir-faire routinisés qu'ils ont intégrés dans l'expérience empirique ordinaire. Le rapport aux objets, l'attention environnementale et la motricité en sont profondément contrariés.

Le rapport aux objets est transformé : les objets virtuels ne fonctionnent pas comme les objets réels. Par exemple, poser/retirer, pour le sens commun, sont des actes inverses. Or dans le cédérom, pour retirer un objet, il faut poser un autre objet sur le premier. Ainsi, le couvercle a posé des problèmes à tous les utilisateurs. En effet on peut mettre le jean dans la cuve alors qu'elle est fermée par le couvercle. On ne peut pas mettre le couvercle si le jean est dans le bain.

Les utilisateurs souffrent d'un déficit sensoriel : on entend bien des bruits de versement lorsque les ingédients tombent dans la cuve, mais, comme une utilisatrice le fait remarquer, on dit dans le cédérom que "les anciens se fiaient à l'odeur et au goût pour évaluer l'évolution du processus chimique" alors qu'aucune indication de ce type n'est donnée à l'utilisateur du cédérom, même sur le mode symbolique.

Des ambiguités entre niveau abstrait et niveau concret sont introduites par le dispositif graphique, qui peut donner à voir un objet là où il n'y a qu'un symbole. Les quantités de produits sont indiquées dans un rectangle, sous forme d'une graduation mathématique. En même temps, lorsqu'on entend les produits s'écouler, le niveau monte dans le rectangle. Au plan visuel, l'écoulement semble se faire vers le haut, ce qui a fortement gêné une utilisatrice qui voyait dans le rectangle un réservoir et ne comprenait pas pourquoi celui-ci se remplissait au lieu de se vider. On constate une fois de plus, à travers cet exemple, un conflit cognitif entre l'interprétation de la symbolisation graphique et les acquis de l'expérience empirique.

Dès lors, l'attention n'est plus canalisée. Lorsque nous sommes dans le monde ordinaire, notre attention est sélective. Des phénomènes inhibiteurs nous permettent de réguler notre accès aux phénomènes en fonction de ce que nous avons déjà intégré comme savoir sur le monde. Dans le monde simulé, l'attention est sursollicitée à plusieurs niveaux simultanément (gestes, lecture du cartouche, voix, icônes). Mis en situation d'incertitude sur la manière de hiérarchiser les informations, l'utilisateur a de la peine à identifier les niveaux de pertinence ou à choisir parmi les éléments redondants. Ainsi nous avons pu constater que la voix masculine qui introduit la manipulation au départ n'était pas écoutée la première fois. Les utilisateurs mobili- 
sent en effet toute leur énergie cognitive pour reconnaître l'environnement graphique. L'observation montre que beaucoup d'entre eux oublient les consignes, pourtant déterminantes, qui sont données à ce moment-là.

La motricité naturelle est en permanence contrariée. Les utilisateurs se focalisent sur les objets à déplacer. Dans la phase de découverte du cédérom, ils oublient les fonctions et s'efforcent d'agir sur les objets avec le seul pointeur. Ils pointent parfois sur le commentaire en voulant déplacer l'objet ou vice versa : désignateur et objet sont pris pour une seule entité, ce qui génère des perturbations. Les choix sémiotiques engendrent des problèmes de "contradictions" dans la finalité des activités motrices. Ainsi, il faut faire glisser avec une main une autre main tenant une baguette pour mélanger. Nous avons alors constaté, chez les utilisateurs, des attitudes compensatoires. Certains, frustrés par la difficulté à faire bouger les objets, font des gestes de la main comme pour saisir des objets à l'écran; certains utilisent le pointeur pour "mélanger" ou pour "tremper".

De manière plus générale, l'utilisateur projette faussement sur ce monde les lois de l'expérience ordinaire. Cela conduit à construire de fausses règles d'action : une utilisatrice s'est efforcée pendant tout le déroulement de l'expérience de remettre le livre en place avec son pointeur. Elle avait d'ailleurs l'impression que son action était efficace et a été surprise quand on lui a montré qu'il était possible de cliquer sur n'importe quelle partie du livre pour qu'il revienne à sa position initiale. Une autre utilisatrice affirme que le pointeur permet de manipuler le jean lors du trempage, alors qu'aucune action n'est possible à ce moment là et qu'en fait le jean bouge tout seul. Mieux, nous avons constaté chez plusieurs personnes une interprétation fantasmée de l'image correspondant à "observer". Le dessus de la cuve apparaît à l'écran. Une voix précise :

S'il n'y a pas de pellicule cuivrée à la surface de l'écume et que le liquide est gris bleu, c'est que la réduction n'est pas assez avancée ou pire, qu'elle est stoppée. Vérifiez la température et le degré d'alcalinité du bain. S'il le faut, relancer la fermentation en ajoutant un peu de mélasse et vérifiez la température du bain.

Trompés par le conditionnel, les utilisateurs tentent de modifier la composition de la mixture puis observent à nouveau. Certains sont 
alors persuadés que le liquide a changé de couleur. Or c'est toujours la même image qui apparaît à l'écran.

\subsection{Construction permanente d'un contexte social fantasmé, chargé d'affects}

Ainsi déstabilisés, les utilisateurs s'interrogent en permanence sur les contraintes de situation ("Qu'est-ce qu'on attend de moi ?")'. Ils procèdent alors, sur la base d'indices analogiques, à des projections de situations sociales ordinaires, qui leur permettent de se positionner.

La projection la plus fréquente est la position "scolaire", vécue tantôt comme positive et tantôt comme négative. Cette position se traduit par un anthropomorphisme conféré à la machine, du reste facilité par l'intervention des voix humaines ou l'introduction de la séquence par un personnage guide, que certains utilisateurs apprécient (c'est une présence, une "petite aide") et que d'autres trouvent insupportable. Dès lors se met en place le sentiment d'entrer dans un dialogue avec un professeur-machine qui est sensé donner des consignes, fournir de l'aide et évaluer. Certains utilisateurs, habitués au contact de l'ordinateur, en sont conscients au début de l'expérience. Mais au fur et à mesure de l'expérience, les aides prennent une forme humaine dans leur inconscient. On constate, lors de leurs interviews, qu'aucun d'entre eux n'utilise les termes "la voix" ou "l'ordinateur". Ils utilisent plutôt un pronom "il(s)" indéterminé. Le plus frappant est le cas des utilisateurs qui ne sont que très peu en contact avec l'ordinateur. Ce sont eux qui "anthropomorphisent" le plus leur relation avec la machine. On pourrait presque établir une relation entre l'habitude de l'ordinateur et la capacité à se décentrer par rapport à la relation homme-machine.

Mais très vite les utilisateurs rejettent l'aide parce qu'elle leur semble inappropriée à leur besoin. Les réponses à leurs attentes sont stéréotypées, figées. Ils le tolèrent au premier essai, puis ils se lassent. Au fur et à mesure des essais (trois en moyenne), ils recourent de moins en moins aux aides sans pour autant réussir. Ainsi que le dit une utilisatrice : 1 Le fait que l'observation se soit déroulée dans un cadre universitaire n'est pas sans
incidence à ce niveau. 
$J$ 'ai écouté les explications la première fois. Mais quand j'ai recommencé les fois suivantes, je n'ai plus écouté parce que c'était toujours la même chose.

En fait, ils attendent un ajustement très souple et permanent à leur conduite que la machine n'est pas en mesure de leur fournir. D'où un sentiment d'agacement.

A un moment, je trouve qu'ils auraient dû dire : "N'oubliez pas votre livre". Non ? Ils ne pouvaient pas rappeler? ... Ils ne voient pas, à force, que la personne elle cherche, elle cherche et que je ne clique pas sur le livre? Ils auraient dû se dire : ben mince ! ... De toutes manières, c'est un ordinateur, hein ! donc...

Chez certains, le sentiment d'être évalué est permanent. Une utilisatrice se comporte exactement comme si elle était "regardée" par l'ordinateur qui jugeait ses actions.

Je me suis dit, ils vont m'interroger sur mes connaissances. Je me suis dit : j'y connais rien. A tous les coups, je ne vais pas savoir répondre.

Ce sentiment de contrôle se double d'un sentiment d'échec. La "pédagogie" que développe le système, du moins au début de la manipulation, procède d'un behaviorisme approximatif qui ne fait que relever les erreurs. Les utilisateurs sont stressés par le bruit "déceptif" qui accompagne les déplacements erronés. Une utilisatrice s'étonne qu'il n'y ait pas de bruit "encourageant" lorsqu'elle s'y prend bien !

Les utilisateurs sont donc mis en demeure de "posséder" ce nouveau monde, sécurisant parce qu'il rappelle le jeu, qu'il est sans risque physique et sans coût économique, mais insécurisant au plan intellectuel parce qu'il donne l'impression qu'on ne le maitrise pas : il faut un effort permanent pour en inférer les règles, trop détachées des savoirs ordinaires. Plus grave, chez certains, l'échec, vécu pourtant par tous les utilisateurs, se traduit par une impression d'insuffisance ou de malaise. Le sentiment de découragement vécu par des adultes en situation expérimentale donne à réfléchir sur l'utilisation de tels produits en milieu scolaire et en situation d'apprentissage. 


\section{Rentabilité cognitive du dispositif}

L'activité inférentielle, parce qu'elle est stimulante au plan intellectuel, suffit-elle en soi à justifier l'usage d'un tel cédérom à des fins d'apprentissage ? Il ne s'agit pas d'un jeu. On n'entre pas dans un monde fictionnel générateur de plaisir ou de poésie. Le monde qui nous est proposé dans la simulation se prétend substituable au nôtre. La simulation dans l'environnement virtuel devrait préparer à l'action dans le monde réel.

\subsection{Un effort cognitif déplacé}

Nous pouvons en fait distinguer trois niveaux de construction de savoirs dans cette expérience :

- un savoir-faire empirique, difficile à formaliser et conjoncturel : comment faire pour agir à l'intérieur de ce cédérom : ex. Je ne peux pas remettre directement un objet à sa place.

- un savoir-faire empirique et transférable concernant la manipulation de l'outil informatique : le pointeur peut changer d'aspect. Il y a une relation entre le mouvement de la souris et le pointeur...

- un savoir scientifique formalisé et transférable : le carbonate de chaux est basique.

Le troisième niveau correspond aux objectifs d'acquisition et devrait donc être l'objet principal de l'effort cognitif. Or pour toutes les observations que nous avons menées, l'investissement majeur des sujets porte sur le premier niveau, celui de la manipulation du cédérom. Lorsque le savoir-faire de niveau 1 est suffisant, l'énergie cognitive des utilisateurs est épuisée et ils renoncent à poursuivre.

La manière dont les savoirs ont été mémorisés est révélatrice des points sur lesquels a porté l'attention des utilisateurs. Or, l'expérience chimique simulée aboutit à une mémorisation "de surface" de type thématique plus que propositionnel. Nous avons demandé aux utilisateurs, juste après l'expérience de dessiner l'écran principal et de reformuler verbalement ce qu'ils avaient retenu. Les utilisateurs retiennent les objets figurant à l'écran et la disposition spatiale de ce dernier. Certains oublient le livre, beaucoup ignorent le cartouche 
(tps/ $/ \% / \mathrm{PH})$ mais dans l'ensemble la mémorisation du dispositif sémiotique est bonne. Par contre, les principes mêmes de l'expérience ne sont pas retenus.

C'est la mémoire visuelle figurative qui a le mieux fonctionné. L'image de l'écran, présente d'un bout à l'autre de l'expérience, s'inscrit précisément dans les représentations : on connaît les ingrédients de la recette, on peut reconstituer un ordre procédural approximatif ; mais les principes de la réaction chimique restent dans l'incertitude. Les indications verbales données par la voix du présentateur ont été consultées de manière aléatoire et très inégale selon les personnes. Leur impact mémoriel est nettement plus faible que celui des informations visuelles. Le livre, par contre, a aidé ceux qui l'ont abondamment consulté. Il ne nous est pas possible pour l'instant de comparer avec certitude le rôle de l'image-écran et le rôle du texte écrit à l'écran dans la mémorisation. Un autre protocole devrait être mis en place pour vérifier ce point.

\subsection{Il est nécessaire de se décentrer et de penser "programmation" pour pouvoir critiquer le produit}

Plus troublant : des utilisateurs "subissent" le produit, et s'avèrent incapables de le critiquer. Ils déplacent alors sur eux-mêmes les défaillances du dispositif.

On pourrait faire appel ici au concept de clarté cognitive, introduit par John Downing ${ }^{1}$ à propos de la lecture des textes. John Downing considère que les lecteurs en grande difficulté n'ont probablement jamais compris ce que représentait le système de l'écrit et plus particulièrement le rapport entre l'écriture et la lecture. Ils ont de la peine à se décentrer et à envisager dans l'écrit la position de "l'autre" qui les sollicite.

Nos observations ont porté sur des utilisateurs qui avait une connaissance très inégale de ce que pouvait être l'informatique. Il en ressort que le sujet ne peut se situer et prendre un recul critique par rapport au dispositif que s'il a une claire conscience de l'existence d'une "couche" de programmation sous-jacente ${ }^{2}$. Il ne s'agit pas

1 J. Downing, J. Fualkow, Lire et raisonner, Toulouse, Privat, 1984.

2 D. CotTe, Sauts technologiques et ruptures dans les modes de représentation des connaissances: étude du texte numérique comme objet technique, Thèse en Sciences de l'Information et de la Communication, Lille 3, 1999. 
d'être capable d'utiliser un langage de programmation, mais d'avoir une représentation de ce qu'est la programmation et de différencier clairement l'interaction homme/machine de l'interaction humaine directe. Cette distinction n'apparaît pas chez tous les sujets que nous avons observés. Son absence peut entraîner un sentiment de malaise.

Ainsi, chez une utilisatrice qui n'a aucune expérience antérieure de l'informatique, on constate un parallélisme flagrant entre un discours inapproprié sur la machine, qu'elle perçoit comme une entité anthropomorphe capable de la voir et de juger ses actions et un vif sentiment d'échec, de culpabilité et d'insuffisance.

Je ne savais pas ce que je devais faire. J'ai été vite découragée. J'avais toujours faux et je ne comprenais pas. Je me suis dit : je suis nulle, quoi, j'en sais rien. Enfin je suis peut-être vite découragée, mais c'est décourageant...

Il existe des degrés dans la représentation qu'on peut avoir de cette "couche" de programmation. Ainsi, des étudiants de maîtrise, qui ont mené une observation d'utilisateurs de ce cédérom, ont eu de la difficulté à dissocier les fonctions différentes associées à chaque objet (Ex. : mélasse (déplacer) / explication sur la mélasse / régler la quantité de mélasse).

Nous-mêmes n'avons pas été suffisamment claires sur ce point. Nous éprouverions maintenant le besoin de confronter nos remarques au point de vue des concepteurs du produit ou d'avoir l'analyse complémentaire d'un informaticien.

\subsection{Mise en évidence de styles cognitifs}

Nous avons en effet retrouvé des polarisations dans les comportements qui relèvent de la psychologie différentielle ${ }^{1}$ :

- caractère auditif ou visuel : certains donnent une place très importante au livre, d'autres préfèrent les explications sonores ;

- tendance à la focalisation ou au balayage : certains parcourent très vite tout l'écran, d'autres explorent minutieusement chaque regroupement de fonctions ;

1 M. HUTEAU, Manuel de psychologie différentielle, Paris, Dunod, 1995. M. REUChLIN, J. LAUTREY et al., Cognition. L'universel et l'individuel, Paris, PUF, 1995. A. DE LA GARANDERIE, Les profils pédagogiques, Paris, Le Centurion, 1980. 
- réflexivité ou impulsivité : certains suivent une démarche méthodique et s'efforcent de contrôler leur action, d'autres se lassent très vite et agissent au jugé pour "voir";

- réaction positive ou négative à la "contrariété" engendrée par le dispositif. Certains le jugent "amusant" et d'autres "perturbant".

Ces différences posent la question de la vicariance ${ }^{1}$, c'est-à-dire du caractère substituable des moyens d'accéder au savoir prévu sur le support. En fait, dans le simulateur de teinture en cuve, les informations concernant l'expérience chimique viennent de canaux différents mais elles se complètent et ne sont donc pas substituables les unes aux autres. Pour réussir, il faut les avoir toutes parcourues. Une forme positive de redondance consisterait à pouvoir réellement choisir le canal d'information.

Le dispositif vise à permettre un apprentissage. Il devrait donc s'adapter, en théorie, aux variations de conduite des individus, à leurs tâtonnements, à leurs erreurs, ce qui suppose que le concepteur envisage la multiplicité des choix que l'utilisateur est potentiellement en mesure d'opérer. Or, il est impossible d'éviter toute rigidité dans la programmation, aussi fine soit-elle.

Il y a là un dilemme pour les concepteurs de produits multimédias. Les utilisateurs ont des profils différents. Il semblerait donc logique de véhiculer la même information par plusieurs canaux différents et de leur laisser le choix. Ce choix peut être conscient ou aléatoire. S'il est aléatoire et par conséquent laissé à l'intuition de l'utilisateur, l'usage devient largement imprévisible : un utilisateur unique peut utiliser plusieurs canaux et trouver insupportable, dans ce cas, un seuil trop élevé de redondance. Pour que le choix soit conscient, il faudrait que l'utilisateur soit en mesure de préciser ses préférences avant d'entrer dans le produit. Alors seulement il serait possible de prévoir un parcours adapté à un type demande sans risquer de fatiguer par le surnombre des redondances. Mais on perdrait alors en "convivialité" en imposant une contrainte à l'utilisateur avant la manipulation à proprement parler. C'est là un équilibre difficile à tenir.

1 P.-Y. GLLLES et al., Psychologie différentielle, Bréal, 1999, pp. 243-245. 


\section{Conclusion : entre un fantôme d'expérience et un ersatz de verbalisation, quel apprentissage ?}

Dans la tradition des apprentissages scientifiques, l'expérience met les élèves en mesure d'observer les phénomènes, développe leur capacité à se poser des questions, leur permet de construire des connaissances par induction, à travers l'action. La verbalisation, avec l'aide d'un enseignant, les amène à exprimer leurs représentations initiales, à les reconsidérer et à s'approprier le savoir savant ; bref, les élèves formalisent la démarche et les connaissances qui en sont le produit.

Dans l'expérience virtuelle qui nous occupe, action et verbalisation sont altérées en profondeur. L'utilisateur du "simulateur de teinture en cuve" est devant une représentation de monde, dans laquelle il agit au moyen de représentations d'actions. Il est canalisé, limité par la programmation qui permet les changements d'état. Il est placé devant un simulacre, plus ou moins réussi, mais, de toute façon, approximatif. Pour maîtriser ce simulacre, il doit désapprendre la réalité et trouver les règles du monde représenté. Ces règles entretiennent un rapport avec les lois qui régissent le monde naturel, mais elles sont simplifiées, raidies.

La déstabilisation qu'induit "l'entrée" dans le produit n'a pas de valeur heuristique par rapport aux contenus scientifiques. Il faut en effet, pour y faire face, construire de nouvelles routines, qui sont uniquement liées à cet environnement particulier. La dépense cognitive nécessaire à la construction des connaissances sur les procédures de l'expérience chimique est en grande partie détournée vers une activité de lecture complexe, déterminée par le support, et nécessitant des savoir-faire très partiellement transférables. De plus, la difficulté que le sujet éprouve à localiser la source de ses échecs le conduit à la lassitude, à la culpabilisation et au malaise.

Si l'on considère, à la suite de Vygotski, que les savoirs informels sont des savoirs qui relèvent de l'expérience empirique et correspondent à un stade préconceptuel, par opposition aux savoirs formels, de niveau scientifique, que l'on peut représenter par un code symbolique, le véritable but de l'apprentissage scientifique devrait se situer au plan des savoirs formels, qu'ils soient d'ordre déclaratif ou d'ordre procédural. Or dans l'expérience de simulation dite interactive 
que nous avons examinée, tout se passe comme si les conflits cognitifs et les efforts déployés par les sujets pour les surmonter se diluaient dans la remise en question permanente des savoirs quotidiens incorporés. Dans ces conditions, le véritable but de l'apprentissage est constamment différé. Le sujet se fatigue, se décourage et finalement s'arrête avant d'avoir appris.

On peut se demander, d'autre part, si l'usage de la machine rend obsolète la présence d'un médiateur humain dans l'apprentissage. En effet, pour que le savoir scientifique puisse se construire de manière stable, il faudrait sans doute passer par une phase verbale qui permette à l'utilisateur de le formaliser. Or, dans la simulation que propose "le secret des couleurs", la verbalisation est présente, mais elle n'est pas le fait de l'utilisateur, qui ne peut pas inscrire sa parole dans le produit. Cependant, 1'observation occasionnelle d'un usage collaboratif de cette séquence nous a montré que la discussion entre pairs (en l'occurrence trois personnes) devant le produit conduit à plus d'attention et à un ajustement plus fin des conduites. Il nous faut poursuivre ce type d'observation pour pouvoir évaluer, en particulier, le degré de mémorisation qui accompagne un usage collectif. Il serait paradoxal, mais intéressant, de découvrir que l'usage le plus efficace d'une telle simulation fût un usage de groupe, alors que "l'individualisation" de l'apprentissage est souvent mise en avant par les enseignants comme le principal avantage que l'on peut retirer des produits numériques.

Ces points nous conduisent à nous interroger sur deux "qualités" attribuées d'ordinaire au multimédia pédagogique : son caractère "ludique" et sa "convivialité", liée à la transparence pour l'utilisateur des aspects informatiques du dispositif. Notre expérience tend à montrer que ces deux traits, valorisés par l'idéologie dominante en matière de nouvelles technologies, peuvent parfois jouer un rôle pervers dans le processus de construction des savoirs. Pour éviter cette réduction idéologique, il convient de définir de nouveaux critères de lisibilité qui permettent d'étalonner les produits non plus seulement en fonction du texte, de l'image et du son, mais aussi du rapport à l'action.

Il faudrait d'autre part, dans des démarches d'apprentissage associées, cette fois, au support, veiller à former des utilisateurs avertis, capables d'évaluer eux-mêmes ce que permet le produit. Cela suppose qu'ils aient une image claire des modes de réalisation des cédéroms et de leurs limites. 